\title{
Rapid Modulation of Sensory Processing Induced by Stimulus Conflict
}

\author{
Lawrence G. Appelbaum, David V. Smith, Carsten N. Boehler, \\ Wen D. Chen, and Marty G. Woldorff
}

\begin{abstract}
Humans are constantly confronted with environmental stimuli that conflict with task goals and can interfere with successful behavior. Prevailing theories propose the existence of cognitive control mechanisms that can suppress the processing of conflicting input and enhance that of the relevant input. However, the temporal cascade of brain processes invoked in response to conflicting stimuli remains poorly understood. By examining evoked electrical brain responses in a novel, hemifield-specific, visualflanker task, we demonstrate that task-irrelevant conflicting stimulus input is quickly detected in higher level executive regions while simultaneously inducing rapid, recurrent modulation of sensory
\end{abstract}

\section{INTRODUCTION}

In our complex and rapidly changing world, humans are continually confronted with environmental stimuli that conflict with task goals and can interfere with successful behavior. The brain's responses to the presence of conflicting or distracting stimuli are thought to include the implementation of cognitive control processes that help to keep attention focused on task-relevant stimuli and to filter out distracting inputs (Egner, 2008; Kerns et al., 2004; Miller \& Cohen, 2001). Indeed, failures or deficiencies of these control processes in response to such distractions can have potentially dire consequences, and their dysfunction is a hallmark of a number of psychopathologies, including depression (Pizzagalli, Peccoralo, Davidson, \& Cohen, 2006), Parkinson's disease (Wylie, Ridderinkhof, Bashore, \& van den Wildenberg, 2009), and schizophrenia (Kerns et al., 2005; Liddle \& Morris, 1991).

Not surprisingly, a number of neuroimaging experiments have investigated the neural responses to the presence of stimulus conflict using such classic stimulus-conflict behavioral paradigms as the Stroop (1935) and Flanker (Eriksen \& Eriksen, 1974) tasks. These neuroimaging studies have implicated a network of regulatory brain areas whose activation increases in the presence of conflict between taskrelevant stimulus input and competing, simultaneously occurring, sensory stimuli (Egner \& Hirsch, 2005; Botvinick,

Duke University, Durham, NC processing in the visual cortex. Importantly, however, both of these effects are larger for individuals with greater incongruencyrelated RT slowing. The combination of neural activation patterns and behavioral interference effects suggest that this initial sensory modulation induced by conflicting stimulus inputs reflects performance-degrading attentional distraction because of their incompatibility rather than any rapid task-enhancing cognitive control mechanisms. The present findings thus provide neural evidence for a model in which attentional distraction is the key initial trigger for the temporal cascade of processes by which the human brain responds to conflicting stimulus input in the environment.

Cohen, \& Carter, 2004; Kerns et al., 2004; Weissman, Giesbrecht, Song, Mangun, \& Woldorff, 2003; Botvinick, Braver, Barch, Carter, \& Cohen, 2001; Casey et al., 2000; MacDonald, Cohen, Stenger, \& Carter, 2000; Botvinick, Nystrom, Fissell, Carter, \& Cohen, 1999). Emerging from this literature has been the idea that the ACC is involved in detecting the presence of stimulus conflict, which then signals strategic control components in the dorsolateral $\mathrm{pFC}$. The $\mathrm{pFC}$ has been proposed to in turn induce a modulatory influence on stimulus processing in the sensory cortices to better manage the conflicting input and respond appropriately (Crump, Vaquero, \& Milliken, 2008; Egner, 2008; Egner, Monti, et al., 2008; Polk, Drake, Jonides, Smith, \& Smith, 2008; Taylor, Nobre, \& Rushworth, 2007; Wendt, Heldmann, Munte, \& Kluwe, 2007; Corballis \& Gratton, 2003).

Nevertheless, these neuroimaging studies of conflictrelated processes have mainly used hemodynamic measures of brain activity, which are substantially limited in temporal resolution (typically $>1 \mathrm{sec}$ ). Moreover, a great many studies have also often focused on analyses of trialto-trial sequential effects related to cognitive control (reviewed in Egner, 2007; Scerif, Worden, Davidson, Seiger, \& Casey, 2006), which would also tend to reflect relatively slow changes over several seconds. On the other hand, those studies that have used higher temporal resolution methods, such as ERPs, have mainly focused on a conflictrelated effect that manifests as a negative-polarity ERP wave over midline frontal sites from 200 to $400 \mathrm{msec}$ (Larson, 
Kaufman, \& Perlstein, 2009; Hanslmayr et al., 2008; Wendt et al., 2007; van Veen \& Carter, 2002; Liotti, Woldorff, Perez, \& Mayberg, 2000). Although this wave has been linked to conflict-related ACC activation observed in neuroimaging studies (Hanslmayr et al., 2008; West, 2003; van Veen \& Carter, 2002), its relationship with any modulations of sensory-cortex processing has been relatively unexplored. Thus, our understanding of the temporal cascade of activity across the various components of these networks in response to the presence of conflicting stimulus input has been rather limited.

To bridge this gap, we used a novel lateralized variant of the Eriksen flanker task (Eriksen \& Eriksen, 1974), in conjunction with the high-temporal resolution electrophysiological methodology of ERPs, to examine rapid and dynamic processes that might occur within a trial in response to conflicting stimulus input. In the typical flanker paradigm, a row of letters or symbols is presented, the central element of which is a target letter to be discriminated, and the flanking letters on the sides are distractors that are either both congruent or both incongruent, with the central target letter. The classic behavioral result observed in such studies is that subjects are slower and less accurate in performing the target letter discrimination when the flankers are incongruent (reviewed in Miller, 1991).

A key, novel feature of the present design was to also include hemifield-specific flanker stimuli that could be congruent on one side of the central target stimulus and incongruent on the other or vice versa. This aspect of the design, combined with the temporal precision of the ERP recordings, allowed us to test whether the presence of conflicting visual stimulus inputs would lead to rapid, spatially specific (i.e., lateralized) modulation of activity in the visual sensory cortices. Using this unique spatial arrangement, it was also possible to disambiguate top-down, sensory-specific attentional modulations in the two hemispheres from more general increases in arousal or effort that might be associated with the processing of stimulus incongruency. Moreover, assuming such rapid sensorycortex modulation was observed, we were interested in whether it would appear to reflect a relative suppression of incongruent stimulus input, consistent with it signaling the rapid instantiation of cognitive control mechanisms for enhancing task performance, or, alternatively, whether the rapid modulation of sensory cortex would be more consistent with reflecting performance-degrading distraction induced by that incongruent input and therefore leading to greater behavioral interference.

\section{METHODS}

\section{Participants}

Sixteen neurologically intact individuals (19-34 years), all with normal or corrected-to-normal visual acuity, participated in this experiment. One subject was excluded from the final analysis due to electrical noise problems in their EEG recordings. All participants gave informed consent before experimentation under a protocol approved by the Duke University Institutional Review Board and were paid $\$ 15 /$ hour. Participants were instructed on the task and given practice experimental runs before the start of the experiment.

\section{Experimental Task}

Each trial consisted of an array of five horizontally arranged letters written in the Lucida Console font and presented $3^{\circ}$ below a central fixation cross for a duration $150 \mathrm{msec}$ (Figure 1). The central letter of each array was designated as the target and consisted of either a "T" or an "I" mapped to response buttons controlled by the index and middle fingers of the right hand, counterbalanced across subjects. Participants were instructed to fixate on the fixation cross, to attend covertly to the central target letter, and to indicate with a button press as rapidly and accurately as possible the identity of the target.

The four lateral letters in the array served as taskirrelevant "flankers" and were to be ignored throughout the experiment. On any given trial, the flankers could
Figure 1. Stimulus schematic. Target letters appeared $3^{\circ}$ below central fixation, surrounded by either bilaterally incongruent flankers, partially incongruent flankers that were incongruent on one side and congruent on the other or bilaterally congruent flankers. The central target letters were either "I"s, as shown here, or "T"s. Stimuli were presented in a random order for $150 \mathrm{msec}$ with a jittered trial-to-trial onset asynchrony interval of 1300 to $1700 \mathrm{msec}$.

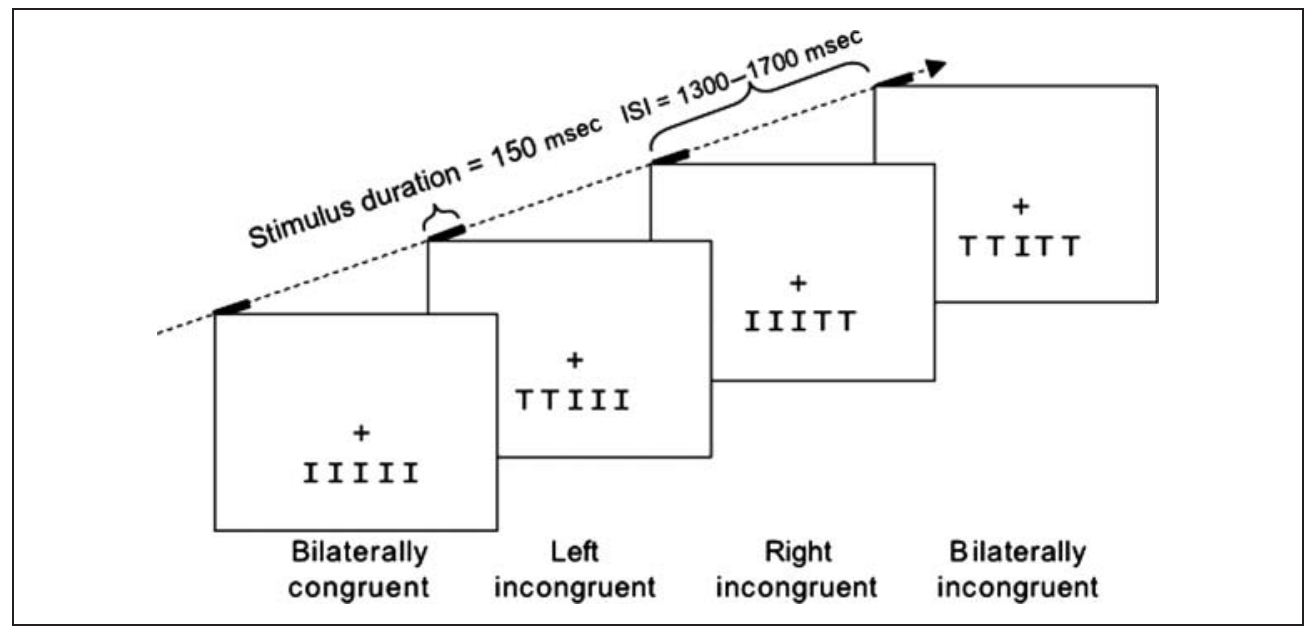


be either all "T"'s or all "I"s and therefore be bilaterally incongruent (I) or congruent (C) with respect to the central target letter. In addition, the flankers could be partially congruent, matching the target letter on one side and not matching on the other side. On left incongruent (LI) trials, the left two flanker letters were incongruent (i.e., mapped to the other response button) with the target letter, whereas the letters on the right were congruent. Conversely, on right incongruent (RI) trials, the right side flankers were incongruent and the left side flankers were congruent.

In total, there were eight stimulus configurations defined by the two targets and four stimulus arrangements: bilaterally congruent (e.g., TTTTT and IIIII), bilaterally incongruent (TTITT and IITII), left incongruent (IITTT and TTIII), and right incongruent (IIITT and TTTII). The total set of trials was composed of $40 \%$ bilaterally congruent, $20 \%$ left incongruent, 20\% right incongruent, and 20\% bilaterally incongruent trials. Individual letters spanned $1.3^{\circ} \times 1.7^{\circ}$ of visual angle with approximately $0.2^{\circ}$ separating each letter. Flankers were located $1.5^{\circ}$ and $3.0^{\circ}$ lateral to the vertical meridian, thereby ensuring that they elicited contralateral processing in visual cortex (Schira, Wade, \& Tyler, 2007). Stimuli were presented in randomized order in a series of experimental runs that lasted roughly 3 min each and consisted of 120 trials. Given the percentages for each trial type noted above, each run thus had 48 bilaterally incongruent trials and 24 each of the left incongruent, right incongruent, and bilaterally incongruent trial types (i.e., the randomization of the trial types was performed without replacement). The timing of the stimulus trials jittered with a random SOA ranging from 1300 to $1700 \mathrm{msec}$, and the contrast of each letter was set to $25 \%$ relative to the background luminance. RTs and error rates (ERs) were monitored while whole head 64-channel EEG was recorded. Before data collection began, participants were given one or two training runs, each consisting of 48 trials, to practice the mapping between the stimuli and the two response buttons as well as practice maintaining central fixation. Data from 16 to 20 runs were collected for each participant, and participants were given the opportunity to rest between runs.

\section{Electrophysiological Data Acquisition}

The EEG was recorded continuously from a 64-channel custom cap (Electro-Cap International, https://www.electrocap.com), using a band-pass filter of 0.01 to $100 \mathrm{~Hz}$ and a sampling rate of $500 \mathrm{~Hz}$ (SynAmps, Neuroscan). All channels were referenced to the right mastoid during recording. The positions of all 64 channels were equally spaced across the customized cap and covered the whole head from slightly above the eyebrows to below the inion (Woldorff et al., 2002). Impedances of all channels were kept below $5 \mathrm{k} \Omega$, and fixation and eye movements were monitored with both EOG recordings and a zoom lens camera. Recordings took place in an electrically shielded, sound-attenuated, dimly lit, experimental chamber.

\section{Behavioral Data Analysis}

Trials were counted as correct if the subject's response occurred within 200 to $1000 \mathrm{msec}$ after presentation of the letter array and corresponded correctly to the target letter. In that no systematic differences were observed for responses to the two different target letters, data were collapsed over the two to arrive at within-participant mean RTs and ERs for each of the four-congruency conditions. Repeated measures ANOVA (rANOVA) and paired $t$ tests were used for statistical analysis, as described by the specific contrasts presented in the Results section. The significance thresholds were set to a $p$ value of .05 and, when applicable, adjusted using the Greenhouse-Geisser correction for nonsphericity.

\section{ERP Analyses}

For each participant, ERPs to the onset of the stimulus array were selectively averaged for each condition. ERP processing included the rereferencing of all channels to the algebraic mean of the two mastoid electrodes and the application of a digital, noncausal, 9-point running average filter, which greatly attenuates signal activity above $56 \mathrm{~Hz}$ at our sample rate of $500 \mathrm{~Hz}$. Artifact rejection of epochs because of blinks, eye movements, or drift was performed off-line using artifact-rejection thresholds that were set individually for each subject, resulting in an average of approximately 12\% of trials being rejected.

We derived individual-subject stimulus-locked averages for correct trials only for each congruency type. To isolate brain potentials related to Flanker interference, three types of difference waves were computed. Full incongruency differences were computed by subtracting the ERPs for congruent trials from incongruent trials (I-C). Partial incongruency differences were derived as the mean of the partial congruency conditions (left and right incongruent) minus congruent trials $((\mathrm{LI}+\mathrm{RI}) / 2-\mathrm{C})$. Finally, lateralized incongruency differences were computed in relation to the side of flanker incongruency by taking the difference between contralateral and ipsilateral incongruent trials for the left and right channels. This difference is analogous to the commonly used N2PC difference (Woodman \& Luck, 1999).

To test for statistically significant differences in the evoked response, rANOVAs were performed over the average response from three ROIs, each comprised of a set of four electrodes. ROIs, shown at the bottom of Figure 3, consisted of the four frontal-central sensors $\mathrm{Cz}, \mathrm{FCz}, \mathrm{Cla}$, and $\mathrm{C} 2 \mathrm{a}$ for the full and partial incongruency differences (solid boxes) and four left posterior sensors (O1i, O1', TO1, and $\mathrm{P} 3 \mathrm{i}$ ) and four right posterior sensors (O2i, O2 ${ }^{\prime}$, TO2, and $\mathrm{P} 4 \mathrm{i}$ ) for the lateralized compatibility differences (dashed boxes). These ROIs were derived from the results of an independent pilot variant of this task collected on 10 subjects and closely corresponded to peaks of the difference wave distributions obtained in the present data 
and in similar tasks reporting a bilateral attention effect to stimuli within the lower visual field (Woldorff et al., 1997, 2002). Additional statistical assessment of the later positive parietal effects occurring between 500 and $650 \mathrm{msec}$ were quantified at channel $\mathrm{Pz}$ (indicated by the shaded gray highlights).

To identify latency ranges at which main effects were significant, ANOVAs were computed in successive 50-msec windows, spanning from 0 to $900 \mathrm{msec}$ relative to a 200 msec prestimulus baseline, for the three incongruency contrasts described above. Latency ranges with greater than three consecutive windows with $p$ values less than .05 were determined to be omnibus significant for a given condition. From these latency ranges, the peak and onset latencies and the peak amplitude values in each ROI and for each congruency contrast were extracted and submitted to between-condition rANOVAs.

To further clarify the relationship between the observed brain activity effects and behavior, subject-wise ERP-behavioral correlations were performed on two of our main contrasts of interest. For full incongruency, the fronto-central incongruent-minus-congruent (I - C) difference wave amplitudes (50-msec windows centered on the peak latency of the respective response) were correlated across subjects with the I - C RT differences. To examine the functional roles of the lateralized occipital incongruency effects, the amplitude difference between the left and the right posterior ROIs for the partial incongruency subtraction (LI-RI) from 400 to $450 \mathrm{msec}$ were correlated with the partial incongruency behavioral interference, measured as the mean RT differences between the partial incongruency trials and the bilaterally congruent trials $(((\mathrm{LI}+\mathrm{RI}) / 2)-\mathrm{C})$.

\section{RESULTS}

In the visual flanker task used here, high-density ERPs were acquired as participants discriminated centrally presented target letters ("I" or "T") that were flanked on the sides with either bilaterally symmetric or laterally asymmetric distractor letters (Figure 1). Consistent with previous behavioral effects in the flanker task (Sanders \& Lamers, 2002; Miller, 1991; Eriksen \& Eriksen, 1974), bilaterally incongruent trials had slower RTs and higher ERs relative to bilaterally congruent trials (incongruent: RT $=537 \mathrm{msec}$, $\mathrm{ER}=12.2 \%$; congruent: RT $=492 \mathrm{msec}, \mathrm{ER}=3.1 \%$; see Figure 2). Importantly, for both RTs and ERs, partially incongruent stimuli resulted in intermediate levels of performance (left: RT $=512 \mathrm{msec}, \mathrm{ER}=5.9 \%$; right: $\mathrm{RT}=$ $513 \mathrm{msec}, \mathrm{ER}=5.5 \%)$. rANOVAs confirmed the presence of significant main effects of stimulus arrangement on both RTs, $F(3,42)=42.01, p<.001$, and Ers, $F(3,42)=$ 23.37, $p<.001$. Subsequent paired $t$ tests revealed significant differences $(p \leq .001)$ in RT and ERs between bilaterally incongruent and bilaterally congruent stimuli and between each of these and the partially incongruent

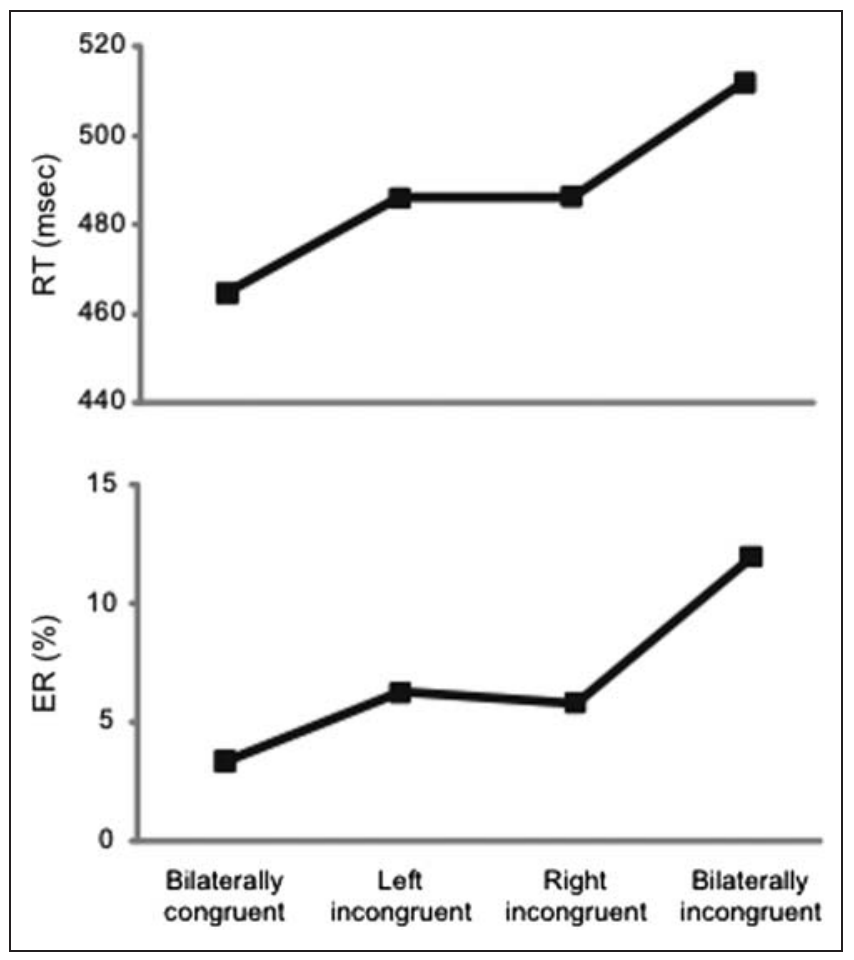

Figure 2. Behavioral results. Mean RTs and ERs showed the greatest interference (slower RTs and more errors) for the incongruent versus congruent conditions, with intermediate levels of interference for the partially incongruent trials.

trial types. There were no significant RT or error-rate differences between left and right conflict trials $(p>.6)$, indicating these elicited comparable levels of behavioral interference.

Having established at the behavioral level that the lateralized incongruent flankers elicited intermediate levels of interference (that were also comparable for the left and right incongruent conditions), we then wished to assess whether these conflicting stimulus inputs could rapidly induce neural effects within the processing of a single trial. To characterize the temporal unfolding of brain processes related to these behavioral effects, we focused on three key ERP contrasts: full incongruency differences (incongruent minus congruent), partial incongruency differences (mean of left and right incongruent minus congruent), and lateralized incongruency differences (contralateral minus ipsilateral to the incongruent side of the partially incongruent trials). The contra-minus-ipsilateral lateralized comparison used here was analogous to the one commonly used for other lateralized functional activations such as the N2pc ERP component that is sensitive to attentional shifting processes (Woodman \& Luck, 1999). More specifically, the responses ipsilateral to the incongruent-flanker side were subtracted from the responses contralateral to the incongruent-flanker side, yielding a continuous measure of contralateralization in the ERP activity across time.

For the above-described functional contrasts, rANOVAs performed over four-electrode sets composing three 
regions-of-interest (ROIs; Figure 3, bottom) were used to test for significant effects across time (see also Figure S1).

As observed in previous studies (Folstein \& Van Petten, 2008; Wendt et al., 2007; van Veen \& Carter, 2002; Heil, Osman, Wiegelmann, Rolke, \& Henninghausen, 2000), flanker incompatibility was reflected by an increased negative-polarity wave (Figure 3A) for bilaterally incongruent versus bilaterally congruent trials over fronto-central electrodes, extending from 150 to $450 \mathrm{msec}$ poststimulus and peaking at around $350 \mathrm{msec}$. Early bilateral activity over the occipital cortex $(<200 \mathrm{msec})$ did not differ for these two conditions and subtracted out (see also Figure S2). This enhanced fronto-central, negative-wave activity in response to incongruent stimuli has been associated with neural sources in ACC and other regions of the frontal cortex (Hanslmayr et al., 2008; van Veen \& Carter, 2002; van Veen, Cohen, Botvinick, Stenger, \& Carter, 2001; Liotti et al., 2000). In addition, as has also been previously reported in ERP tasks using stimulus conflict (Appelbaum, Meyerhoff, \& Woldorff, 2009; Larson et al., 2009; Atkinson, Drysdale, \& Fulham, 2003; Liotti et al., 2000; West \& Alain, 1999), a second, later, significant difference was also observed between 500 and $650 \mathrm{msec}$ (gray shaded) in which incongruent trials elicited a stronger positive-polarity deflection over parieto-occipital sites.
As seen in the full incongruency differences, the partial incongruency trials relative to the full-congruency trials also evoked a very similar, albeit somewhat reduced, frontocentral, negative-polarity wave from 200 to $400 \mathrm{msec}$ (peak amplitude $-2.3 \mu \mathrm{V}$ vs. $-1.6 \mu \mathrm{V} ; t=-2.53, p=.024$ ), which was also followed by a similar but smaller parietal positivity (Figure 3B). This result indicates that the interference induced by the partially incongruent flankers was reflected by an intermediate level of activity in the same conflictprocessing fronto-parietal cortical network and closely paralleled the intermediate level of behavioral incongruency effects observed for these trial types.

As indicated by the behavioral performance, the lateralized partially incongruent conditions shared a common net level of behavioral interference but were designed such that directly contrasting the associated ERP responses for these two conditions would isolate any effects specific to the spatial arrangement of the asymmetric incongruency within the two visual hemifields. As hypothesized, the contralateral versus ipsilateral incongruency contrast did indeed result in significant, opposite-polarity potentials over the occipital cortices (peak amplitude left $=-1.3 \mu \mathrm{V}$, right $=0.8 \mu \mathrm{V} ; t=-7.08, p<.001)$, beginning at around $200 \mathrm{msec}$ and peaking at around $400 \mathrm{msec}$ (Figure 3C). These occipital lateralized incongruency effects began at

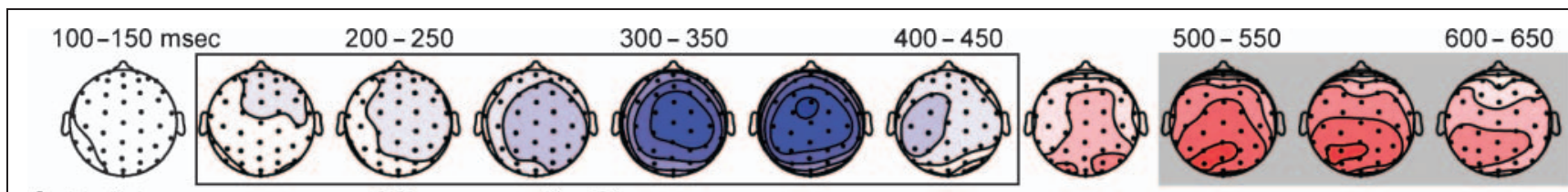

A Full incongruency difference: (I-C)

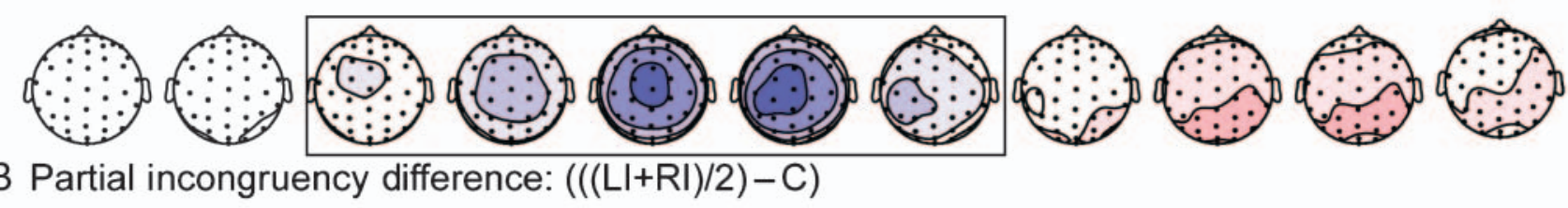

\section{B Partial incongruency difference: $(((\mathrm{LI}+\mathrm{RI}) / 2)-\mathrm{C})$}

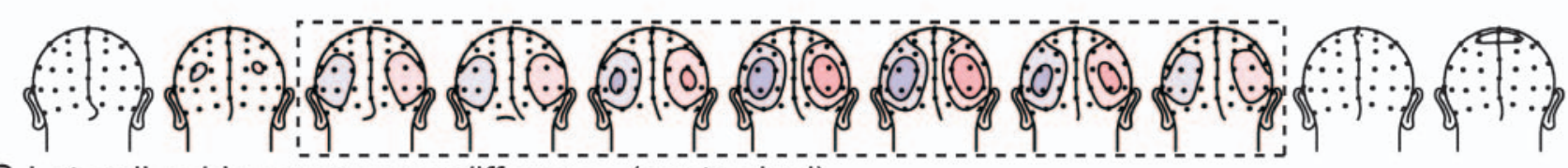

\section{Lateralized incongruency difference (contra-ipsi)}
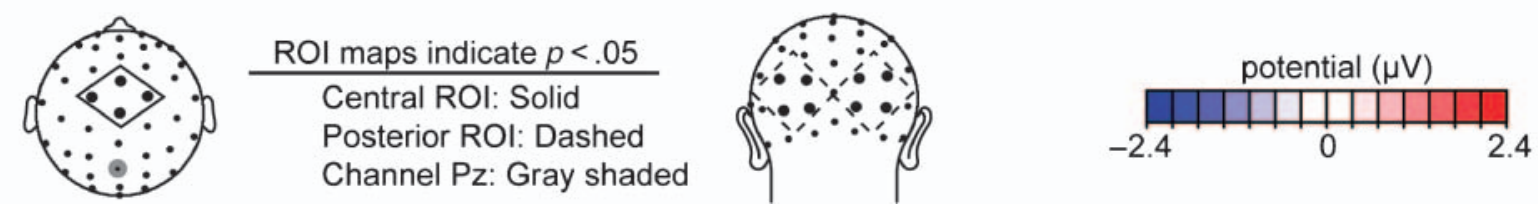

Figure 3. Difference wave topographic distributions across time for the various key functional contrasts. (A) Bilaterally incongruent minus bilaterally congruent trials; (B) mean of partially incongruent trials minus bilaterally congruent trials; and (C) contralateral minus ipsilateral incongruent trials (contra-minus-ipsi on the right, ipsi-minus-contra on the left). Each map is the activity difference averaged over 50 msec, presented from a top view for the full and partial incongruency differences (A, B) and from a posterior view for the lateralized incongruency difference $(C)$. Maps that reached statistical significance $(p<.05)$, according to rANOVAs performed over the central (solid), posterior (dashed), and $\mathrm{Pz}$ (gray) electrode ROIs that are indicated by gray bars running across time and surrounding the maps. The location of these ROIs is indicated in the legend below. 
Figure 4. Relationships between incongruency-related RT and ERP effects.

(A) Individual-subject RT and fronto-central ERP effect values for the full incongruency contrasts, along with the linear regression fit lines. (B) Same as panel A, but for the RT effects and lateralized-occipital ERP effects for the partially incongruency conditions. In both cases, greater behavioral interference correlated with greater voltage differences in the ERP

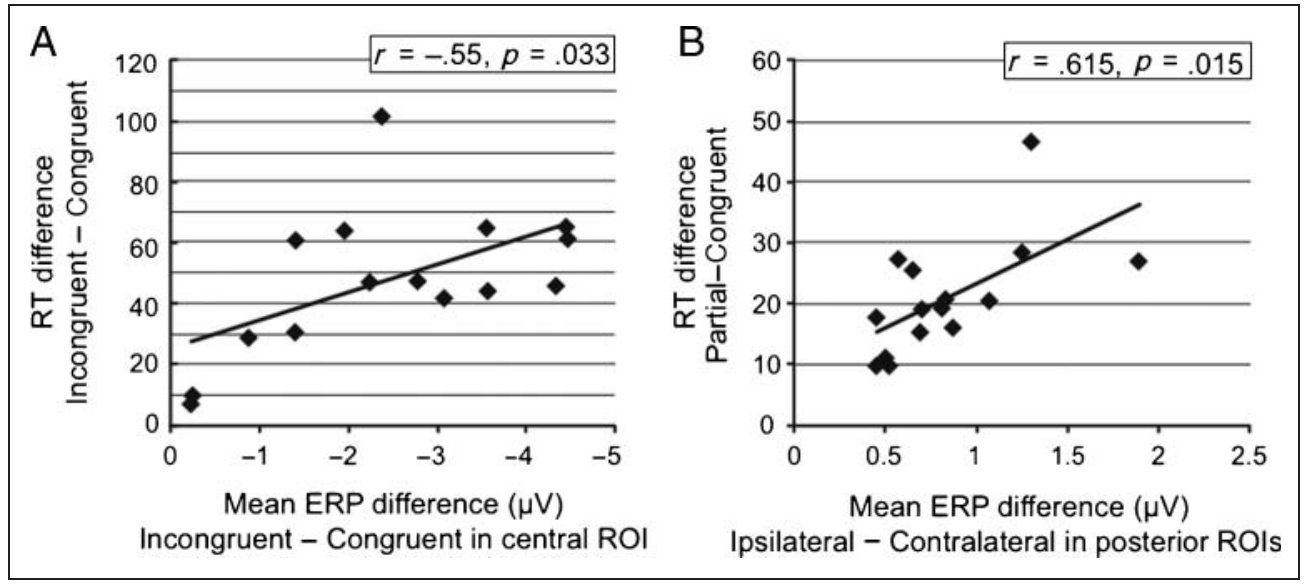

around the same time as the fronto-central incongruency effects and grew in parallel with them across several hundred milliseconds, with neither the peak nor the onset latencies differing significantly between the two. The lateralized occipital effects were generally more pronounced in the left than the right ROI $(p=.013)$, especially at the earliest latencies, consistent with previous behavioral (Weekes \& Zaidel, 1996) and neuroimaging (Spencer \& Banich, 2005) studies that report left hemisphere bias in the response strength to incompatibilities in the two visual fields.

To further assess the relationship between these ERP components and behavior, we computed subject-wise Pearson's correlation coefficients between the incongruency RTs and ERP effects. These comparisons (Figure 4) revealed significant correlations between the RT slowing on bilaterally incongruent trials (relative to bilaterally congruent) and the corresponding full incongruency ERP effects $(r=-.552, p=.033)$ as well as between the partial incongruency RT slowing (also relative to bilaterally congruent) and the partial incongruency lateralized occipital ERP difference $(r=.615, p=.015)$. In both cases, greater ERP difference amplitudes correlated with greater RT slowing. These correlational results thus support a functional relationship between task performance during the visual conflict paradigm and fronto-central and occipital neural activity patterns.

\section{DISCUSSION}

The present results demonstrate for the first time the occurrence of rapid, spatially selective modulation of activity in the visual cortex induced by the presence of stimulus conflict. In the initial feed-forward sweep of activity, the early sensory responses in visual cortex (P1 and N1 sensory ERP components, $<175 \mathrm{msec}$ ) did not show any influence of the presence or location of the stimulus incompatibility. However, spatially specific, conflict-related modulation of the activity in these sensory areas was observed a short time later, initiating at approximately $200 \mathrm{msec}$ and lasting several hundred milliseconds. This rapid sensory-cortex adjustment, revealed by the hemifield-specific arrangement of conflicting versus nonconflicting visual stimuli, occurred concurrently with the spatially nonspecific, negative-polarity ERP effect of stimulus incongruency over higher level executive control areas in the frontal cortex (Folstein \& Van Petten, 2008; van Veen \& Carter, 2002; Heil et al., 2000). The temporal co-occurrence from 200 to $500 \mathrm{msec}$ of the fronto-central incongruency effects and the lateralized incongruency-related modulations of the visual sensory cortex suggest that these processes build up in concert, perhaps through an evolving flow of dynamic interactions across this period.

Individual differences in the amplitude of both of these incongruency-related ERP effects correlated with the size of the incongruency RT effects, suggesting a functional interpretation of the brain's response to stimulus conflict under these conditions. Consistent with some previously reported N2 (Folstein \& Van Petten, 2008) and N450 (Larson et al., 2009) conflict-induced ERP effects, individual differences in the amplitude of the fronto-central incongruency ERP effects correlated positively with the size of the RT effects (i.e., with greater RT slowing). In addition, however, the contemporaneous, lateralized occipital ERP effect revealed in this study was also larger for subjects with greater RT slowing. The direction of these correlations suggests that these neural effects reflect increased interference or distraction caused by the incompatible components of the stimulus input rather than suppressive processes that would have served to enhance task performance and thus would have been expected to correlate with reduced RT slowing (however, see Wager et al., 2005, for a cautionary discussion regarding the interpretation of positive correlations in putative interference-resolution mechanisms).

More specifically, we interpret the increased lateralized occipital effects in the partially incongruent trials as resulting from increased distraction by and thus attention toward the incongruent flankers. On the basis of numerous previous spatial attention studies (reviewed in Hillyard, Mangun, Woldorff, \& Luck, 1995), such a result would be expected to be reflected neurophysiologically by increased 
differences in ERP amplitude between the two sides of the visual cortex, as we observed. In accordance, this pattern of increased distraction by the incongruent stimuli was accompanied by corresponding subject-wise increases in RT slowing. In contrast, if the increased, early latency, lateraloccipital asymmetry was actually representative of greater suppression of the incongruent flankers and/or greater relative processing of the congruent flankers, then the amplitude of the laterally asymmetric occipital ERP effects should have correlated with reduced RT slowing, rather than the increased slowing that was observed.

Importantly, as noted above, the increasing lateralized occipital effect over the 200- to 500-msec period was accompanied by an increasing, spatially nonspecific, main effect of incongruency over the fronto-central scalp over the same period. These two effects did not show any definitive sequence of occurrence in which one clearly preceded the other. Rather, these components begin at about the same time and showed no significant differences in their onset or even peak latencies, with both growing in strength, in parallel, over the same several hundred-millisecond period. One potential explanation for this parallel-increasing activation pattern is that as attention was initially drawn toward the incongruent distractors, the increased processing of those distractors delivered increased conflicting input to the higher level regions in frontal cortex that detect such conflict (e.g., ACC), leading to a corresponding rise in activity in these regions and contributing even further to the slowing of the RT. This steadily increasing detection of the conflicting stimulus input may have then led to yet more attentional distraction toward those conflicting elements, leading to yet more lateralized-asymmetric activation in the visual cortex and yet more RT slowing. These fronto-occipital interaction processes may therefore have fed into each other, creating increasing parallel frontal and occipital effects across several hundred milliseconds.

It is possible, perhaps even likely, that the initial, rapid, attentional distraction caused by the incongruent flankers identified here is followed in time by the invocation of cognitive control processes that can help deal with stimulus conflict. In particular, it has been proposed that such cognitive control processes might include the enhancement of processing in the sensory cortices of the task-relevant stimulus input or the suppression of the distracting input brought about by networks that include ACC and the lateral pFC (for reviews, see Mansouri, Tanaka, \& Buckley, 2009; Egner, 2008; Botvinick et al., 2004; Ridderinkhof, Ullsperger, Crone, \& Nieuwenhuis, 2004). The possible occurrence of such control processes at longer latencies may be the source of previously reported conflict-related modulatory effects in fMRI experiments, including betweentrial adaptation effects (Egner, Monti, et al., 2008; Polk et al., 2008; Egner \& Hirsch, 2005; Badre \& Wagner, 2004; Kerns et al., 2004; Weissman et al., 2003; Bunge, Hazeltine, Scanlon, Rosen, \& Gabrieli, 2002; Casey et al., 2000; Hazeltine, Poldrack, \& Gabrieli, 2000; MacDonald et al., 2000; Botvinick et al., 1999). It may be, however, that not only do such modulatory effects occur at longer latencies but also across a longer duration of time, making them more likely to be picked up by the much slower signal of fMRI that integrates activity across time. Moreover, fMRI may be insensitive to the initial, rapid effects shown here, or it may be that these early distraction-related effects are swamped out in the fMRI signal by later, longerlasting, adjustments in the opposite direction, with the fMRI not having the temporal resolution to be able to disentangle the two. Regardless, however, any such slower, hemodynamically based measures that may detect neural activity variations occurring across substantially longer time frames would not seem to be sensitive to the rapid conflict-related modulations of sensory processing revealed in this study.

From a broader view, however, subjects usually do respond correctly, albeit more slowly, on the incongruent trials of these stimulus-conflict paradigms, even in the face of the attentional distraction toward the incongruent stimulus components. Such a pattern of results might be explained by the operation of a control mechanism at the level of response selection and execution. For example, in a recent flanker study combining ERPs and TMS (Taylor et al., 2007), the ERP component known as the lateralized readiness potential was used as a gauge of the responselevel effects of stimulus conflict. The study reported that stimulus conflict modulated processing at the response preparation phase of incongruent trials (see also Gratton, Coles, \& Donchin, 1992), consistent with the slower RTs observed on these trials, with TMS-induced disruption of dorsal-medial-frontal cortex indicating that these response-level effects were modulated by input from frontal brain regions. The findings in this study are not inconsistent with such results, however. Specifically, if attention is distracted toward incongruent stimulus input during the course of a trial, as evidenced by the rapid sensory effects observed here, then the frontal cortex-mediated adjustments of response processes may well reflect attempts to overcome such attentional distraction by slowing up the output phase to help ensure the correct behavioral response (Ridderinkhof, van den Wildenberg, Wijnen, \& Burle, 2004). Indeed, the early attentional distraction processes revealed in this study may feed into and help induce the modulation of response processes by the frontal cortex. These response-level adjustments may in turn be followed by task-enhancing modulation of sensoryprocessing activity at longer latencies, either for the next trial in an experimental paradigm (e.g., Scerif et al., 2006) or for the next phase of behavior in real life.

The ways in which the brain responds to conflicting or distracting stimulus input in our complex world are critical to successful behavior. Previous imaging studies have suggested the existence of cognitive control adjustments in response to conflicting stimulus input that include suppression of the conflicting input and/or enhancement of the task-relevant input. In this study, high-temporal resolution electrophysiological recordings, along with the 
hemifield-specific arrangement of the incongruent stimulus input, provide direct neural evidence that the initial modulation of sensory cortex activity induced by conflicting stimulus input reflects the task performance-degrading distractibility of the incongruent input rather than a task-enhancing sensory-processing modulation. This incongruency-induced distraction may then be followed by and indeed may help induce later task-enhancing cognitive control mechanisms that have been reported with fMRI. Nonetheless, the present findings provide neural evidence for a model in which attentional distraction is the key initial trigger for the temporal cascade of processes by which the human brain responds to conflicting stimulus input in their environment. Moreover, the present results suggest that those individuals who are able to set up a strong selective attention filter at the front end to focus on the task-relevant input and filter out the irrelevant are less impaired by stimulus conflict. These findings therefore provide new insights into the rapid and dynamic mechanisms by which the human brain responds to conflicting stimuli that can distract from behavioral goals.

\section{Acknowledgments}

This work was supported by grant nos. R01-MH60415 and R01NS051048 from the National Institutes of Health to MGW. The authors thank Tobias Egner for his helpful comments on this manuscript and Rob Won and Lauren Davis for their help with data collection.

Reprint requests should be sent to Lawrence G. Appelbaum, Duke University, Box 90999, Durham NC, 27708, or via e-mail: greg@ duke.edu.

\section{REFERENCES}

Appelbaum, L. G., Meyerhoff, K. L., \& Woldorff, M. G. (2009). Priming and backward influences in the human brain: Processing interactions during the Stroop interference effect. Cerebral Cortex, 19, 2508-2521.

Atkinson, C. M., Drysdale, K. A., \& Fulham, W. R. (2003). Event-related potentials to Stroop and reverse Stroop stimuli. International Journal of Psychophysiology, 47, $1-21$.

Badre, D., \& Wagner, A. D. (2004). Selection, integration, and conflict monitoring; assessing the nature and generality of prefrontal cognitive control mechanisms. Neuron, 41, 473-487.

Botvinick, M., Nystrom, L. E., Fissell, K., Carter, C. S., \& Cohen, J. D. (1999). Conflict monitoring versus selection-for-action in anterior cingulate cortex. Nature, 402, 179-181.

Botvinick, M. M., Braver, T. S., Barch, D. M., Carter, C. S., \& Cohen, J. D. (2001). Conflict monitoring and cognitive control. Psychological Review, 108, 624-652.

Botvinick, M. M., Cohen, J. D., \& Carter, C. S. (2004). Conflict monitoring and anterior cingulate cortex: An update. Trends in Cognitive Sciences, 8, 539-546.

Bunge, S. A., Hazeltine, E., Scanlon, M. D., Rosen, A. C., \& Gabrieli, J. D. (2002). Dissociable contributions of prefrontal and parietal cortices to response selection. Neuroimage, 17, $1562-1571$.

Casey, B. J., Thomas, K. M., Welsh, T. F., Badgaiyan, R. D., Eccard, C. H., Jennings, J. R., et al. (2000). Dissociation of response conflict, attentional selection, and expectancy with functional magnetic resonance imaging. Proceedings of the National Academy of Sciences, U.S.A., 97, 8728-8733.

Corballis, P. M., \& Gratton, G. (2003). Independent control of processing strategies for different locations in the visual field. Biological Psychology, 64, 191-209.

Crump, M. J., Vaquero, J. M., \& Milliken, B. (2008). Context-specific learning and control: The roles of awareness, task relevance, and relative salience. Consciousness and Cognition, 17, 22-36.

Egner, T. (2007). Congruency sequence effects and cognitive control. Cognitive, Affective \& Behavioral Neuroscience, 7, 380-390.

Egner, T. (2008). Multiple conflict-driven control mechanisms in the human brain. Trends in Cognitive Sciences, 12, 374-380.

Egner, T., \& Hirsch, J. (2005). Cognitive control mechanisms resolve conflict through cortical amplification of task-relevant information. Nature Neuroscience, 8, 1784-1790.

Egner, T., Monti, J. M., Trittschuh, E. H., Wieneke, C. A., Hirsch, J., \& Mesulam, M. M. (2008). Neural integration of top-down spatial and feature-based information in visual search. Journal of Neuroscience, 28, 6141-6151.

Eriksen, B. A., \& Eriksen, C. W. (1974). Effects of noise letters upon the identification of a target letter in a nonsearch task. Perception \& Psychophysics, 16, 143-149.

Folstein, J. R., \& Van Petten, C. (2008). Influence of cognitive control and mismatch on the N2 component of the ERP: A review. Psychophysiology, 45, 152-170.

Gratton, G., Coles, M. G., \& Donchin, E. (1992). Optimizing the use of information: Strategic control of activation of responses. Journal of Experimental Psychology: General, 121, 480-506.

Hanslmayr, S., Pastotter, B., Bauml, K. H., Gruber, S., Wimber, M., \& Klimesch, W. (2008). The electrophysiological dynamics of interference during the Stroop task. Journal of Cognitive Neuroscience, 20, 215-225.

Hazeltine, E., Poldrack, R., \& Gabrieli, J. D. (2000). Neural activation during response competition. Journal of Cognitive Neuroscience, 12(Suppl. 2), 118-129.

Heil, M., Osman, A., Wiegelmann, J., Rolke, B., \& Henninghausen, E. (2000). N200 in the Eriksen-task: Inhibitory executive processes? Journal of Psychophysiology, 14, 218-225.

Hillyard, S. A., Mangun, G. R., Woldorff, M. G., \& Luck, S. J. (1995). Neural systems mediating selective attention. In M. S. Gazzaniga (Ed.), Handbook of cognitive neuroscience (pp. 665-681). Cambridge, MA: MIT Press.

Kerns, J. G., Cohen, J. D., MacDonald, A. W., III, Cho, R. Y., Stenger, V. A., \& Carter, C. S. (2004). Anterior cingulate conflict monitoring and adjustments in control. Science, 303, 1023-1026.

Kerns, J. G., Cohen, J. D., MacDonald, A. W., III, Johnson, M. K., Stenger, V. A., Aizenstein, H., et al. (2005). Decreased conflict- and error-related activity in the anterior cingulate cortex in subjects with schizophrenia. American Journal of Psychiatry, 162, 1833-1839.

Larson, M. J., Kaufman, D. A., \& Perlstein, W. M. (2009). Neural time course of conflict adaptation effects on the Stroop task. Neuropsychologia, 47, 663-670.

Liddle, P. F., \& Morris, D. L. (1991). Schizophrenic syndromes and frontal lobe performance. British Journal of Psychiatry, $158,340-345$.

Liotti, M., Woldorff, M. G., Perez, R., \& Mayberg, H. S. (2000). An ERP study of the temporal course of the Stroop color-word interference effect. Neuropsychologia, 38, 701-711. 
MacDonald, A. W., III, Cohen, J. D., Stenger, V. A., \& Carter, C. S. (2000). Dissociating the role of the dorsolateral prefrontal and anterior cingulate cortex in cognitive control. Science, 288, 1835-1838.

Mansouri, F. A., Tanaka, K., \& Buckley, M. J. (2009). Conflict-induced behavioural adjustment: A clue to the executive functions of the prefrontal cortex. Nature Reviews Neuroscience, 10, 141-152.

Miller, E. K., \& Cohen, J. D. (2001). An integrative theory of prefrontal cortex function. Annual Review of Neuroscience, 24, 167-202.

Miller, J. (1991). The flanker compatibility effect as a function of visual angle, attentional focus, visual transients, and perceptual load: A search for boundary conditions. Perception \& Psychophysics, 49, 270-288.

Pizzagalli, D. A., Peccoralo, L. A., Davidson, R. J., \& Cohen, J. D. (2006). Resting anterior cingulate activity and abnormal responses to errors in subjects with elevated depressive symptoms: A 128-channel EEG study. Human Brain Mapping, 27, 185-201.

Polk, T. A., Drake, R. M., Jonides, J. J., Smith, M. R., \& Smith, E. E. (2008). Attention enhances the neural processing of relevant features and suppresses the processing of irrelevant features in humans: A functional magnetic resonance imaging study of the Stroop task. Journal of Neuroscience, 28, 13786-13792.

Ridderinkhof, K. R., Ullsperger, M., Crone, E. A., \& Nieuwenhuis, S. (2004). The role of the medial frontal cortex in cognitive control. Science, 306, 443-447.

Ridderinkhof, K. R., van den Wildenberg, W. P. M., Wijnen, J., \& Burle, B. (2004). Response inhibition in conflict tasks is revealed in delta plots. In M. I. Posner (Ed.), Cognitive neuroscience of attention (pp. 369-377). New York: Guilford Press.

Sanders, A. F., \& Lamers, J. M. (2002). The Eriksen flanker effect revisited. Acta Psychologica (Amsterdam), 109, $41-56$.

Scerif, G., Worden, M. S., Davidson, M., Seiger, L., \& Casey, B. J. (2006). Context modulates early stimulus processing when resolving stimulus-response conflict. Journal of Cognitive Neuroscience, 18, 781-792.

Schira, M. M., Wade, A. R., \& Tyler, C. W. (2007). Two-dimensional mapping of the central and parafoveal visual field to human visual cortex. Journal of Neurophysiology, 97, 4284-4295.

Spencer, K. M., \& Banich, M. T. (2005). Hemispheric biases and the control of visuospatial attention: An ERP study. BMC Neuroscience, 6, 51.

Stroop, J. R. (1935). Studies of interference in serial verbal reactions. Journal of Experimental Psychology, 18, 643-662.
Taylor, P. C., Nobre, A. C., \& Rushworth, M. F. (2007). Subsecond changes in top-down control exerted by human medial frontal cortex during conflict and action selection: A combined transcranial magnetic stimulation electroencephalography study. Journal of Neuroscience, 27, 11343-11353.

van Veen, V., \& Carter, C. S. (2002). The anterior cingulate as a conflict monitor: fMRI and ERP studies. Physiology E Behavior, 77, 477-482.

van Veen, V., Cohen, J. D., Botvinick, M. M., Stenger, V. A., \& Carter, C. S. (2001). Anterior cingulate cortex, conflict monitoring, and levels of processing. Neuroimage, 14 , 1302-1308

Wager, T. D., Sylvester, C. Y., Lacey, S. C., Nee, D. E., Franklin, M., \& Jonides, J. (2005). Common and unique components of response inhibition revealed by fMRI. Neuroimage, 27, 323-340.

Weekes, N. Y., \& Zaidel, E. (1996). The effects of procedural variations on lateralized Stroop effects. Brain and Cognition, 31, 308-330.

Weissman, D. H., Giesbrecht, B., Song, A. W., Mangun, G. R., \& Woldorff, M. G. (2003). Conflict monitoring in the human anterior cingulate cortex during selective attention to global and local object features. Neuroimage, 19, 1361-1368.

Wendt, M., Heldmann, M., Munte, T. F., \& Kluwe, R. H. (2007). Disentangling sequential effects of stimulus- and response-related conflict and stimulus-response repetition using brain potentials. Journal of Cognitive Neuroscience, 19, 1104-1112.

West, R. (2003). Neural correlates of cognitive control and conflict detection in the Stroop and digit-location tasks. Neuropsychologia, 41, 1122-1135.

West, R., \& Alain, C. (1999). Event-related neural activity associated with the Stroop task. Brain Research, Cognitive Brain Research, 8, 157-164.

Woldorff, M. G., Fox, P. T., Matzke, M., Lancaster, J. L., Veeraswamy, S., Zamarripa, F., et al. (1997). Retinotopic organization of early visual spatial attention effects as revealed by PET and ERPs. Human Brain Mapping, 5, 280-286.

Woldorff, M. G., Liotti, M., Seabolt, M., Busse, L., Lancaster, J. L., \& Fox, P. T. (2002). The temporal dynamics of the effects in occipital cortex of visual-spatial selective attention. Brain Research, Cognitive Brain Research, 15, 1-15.

Woodman, G. F., \& Luck, S. J. (1999). Electrophysiological measurement of rapid shifts of attention during visual search. Nature, 400, 867-869.

Wylie, S. A., Ridderinkhof, K. R., Bashore, T. R., \& van den Wildenberg, W. P. (2009). The effect of Parkinson's disease on the dynamics of on-line and proactive cognitive control during action selection. Journal of Cognitive Neuroscience, 22, 2058-2073. 